\title{
Effects of oral administration of brassica secondary metabolites, allyl cyanide, allyl isothiocyanate and dimethyl disulphide, on the voluntary food intake and metabolism of sheep
}

\author{
BY ALAN J. DUNCAN AND JOHN A. MILNE \\ Macaulay Land Use Research Institute, Craigiebuckler, Aberdeen AB 9 2QJ
}

(Received 19 February 1992 - Accepted 17 October 1992)

\begin{abstract}
Glucosinolates, such as sinigrin, and S-methyl cysteine sulphoxide (SMCO), which are found in forage brassica species have been implicated in the low intakes observed among lambs consuming such diets. To test both the individual and interactive effects of these compounds in sheep, all combinations of the sinigrin breakdown products, allyl cyanide (ACN) and allyl isothiocyanate (AITC; $10 \mathrm{mmol} / \mathrm{d}$ ), and the SMCO metabolite dimethyl disulphide (DMDS; $25 \mathrm{mmol} / \mathrm{d}$ ) were orally administered twice daily for 5 weeks to forty sheep offered dried grass pellets ad lib. As well as measuring voluntary food intake (VFI), a number of haematological and clinical function tests were conducted to assess the physiological effects of the compounds. VFI was significantly depressed by both ACN and AITC but not by DMDS. DMDS significantly ameliorated the effects of $A C N$ on VFI $(P<0.001)$. Concentrations of reduced glutathione in the blood were depressed by ACN and AITC and elevated by DMDS but no significant interactions were evident. Elevated plasma $\gamma$-glutamyl transpeptidase (EC 2.3.2.1) activity on ACN and AITC treatments indicated possible liver damage. DMDS elicited a rise in Heinz bodies to $11 \%$ by week 2 but this was not reflected in packed cell volume and blood haemoglobin levels which were unaffected by treatment. The increased Heinz body count caused by DMDS was not further influenced by ACN or AITC. In conclusion, the depressive effects of sinigrin breakdown products on VFI were not compounded by the additional presence of DMDS which, on the contrary, lessened the depression of VFI caused by ACN.
\end{abstract}

Glucosinolate breakdown products: Voluntary food intake: Brassica species: Lambs

Forage brassica species are widely used in temperate agricultural systems as a feed for ruminants. These species contain representatives of two groups of plant secondary compounds which have potentially harmful effects to ruminants. Glucosinolates are a group of plant thioglucosides which undergo hydrolysis when the vegetative parts of the plant are damaged to yield a range of potentially toxic compounds. These include nitriles, isothiocyanates, 5-vinyl oxazolidine-2-thione and thiocyanate ions (Fenwick \& Heaney, 1983). S-methyl cysteine sulphoxide (SMCO) is a non-protein amino acid whose rumen metabolite, dimethyl disulphide (DMDS), causes haemolytic anaemia in ruminants (Smith, 1974).

The range of products arising as a result of glucosinolate hydrolysis is dependent on the chemical environment in which hydrolysis occurs as well as the identity of the parent glucosinolates (Fenwick \& Heaney, 1983). For example, low pH tends to favour nitrile production, with increasing $\mathrm{pH}$ leading to greater production of isothiocyanates. Furthermore, sinigrin hydrolysis yields allyl cyanide (ACN) as the nitrile metabolite while the higher homologue, gluconapin, yields butenyl cyanide. Different nitrile or isothiocyanate compounds differ in their toxicity as a result of their different disposition following ingestion (Silver et al. 1982; Mennicke et al. 1983). Toxicity, however, is almost 
certainly associated with the presence of the $\mathrm{C} \equiv \mathrm{N}$ or $\mathrm{N}=\mathrm{C}=\mathrm{S}$ groups and inferences can be drawn on the mechanisms of toxicity, if not the magnitude of toxicity of particular compounds, from findings on related compounds.

The toxicity of glucosinolate-derived nitriles is generally attributed to their effects on the liver and kidney (VanEtten et al. 1969; Srivastava et al. 1975). A number of histological studies in non-ruminants have demonstrated cellular damage in liver and kidney tissues following nitrile administration (Nishie \& Daxenbichler, 1980; Gould et al. 1985). Another potential route of nitrile toxicity is suppression of cellular respiration following release of free cyanide in the tissues and consequent inhibition of cytochrome oxidase $(E C$ 1.9.3.1) activity (Ohkawa et al. 1972; Willhite \& Smith, 1981; Ahmed \& Farooqui, 1982; Tanii \& Hashimoto, 1984). The toxic effects of isothiocyanates include effects on thyroid function (Langer, 1964; Langer \& Stolc, 1965), but more direct toxic effects may arise as a result of their extremely electrophilic nature and their capacity to bind the sulphydryl groups of biologically important molecules (Bjorkman, 1973; Tang, 1974; Kawakishi \& Namiki, 1982; Kawakishi et al. 1983). The toxic importance of such reactions has yet to be demonstrated in vivo.

Following absorption and entry into the peripheral circulation, DMDS disrupts erythrocyte glutathione metabolism (Steven et al. 1981). As a result, the mechanisms which normally protect erythrocytes from oxidative injury caused by release of oxygen radicals and peroxides in the normal metabolism of the erythrocyte (Gordon-Smith \& White, 1974) are inhibited, leading ultimately to cell lysis and haemolytic anaemia.

The potential interaction between SMCO and glucosinolates in producing detrimental effects in ruminants has been suggested (Smith, 1980), but the only evidence comes from experiments in which artificial administration of either glucosinolates or SMCO resulted in more severe effects when the compounds were given to animals already consuming a brassica diet (Barry et al. 1982; Duncan, 1990). In order to investigate, in ruminants, the main physiological effects as well as potential interactions between secondary compounds found in brassicas, an experiment was conducted in which sheep were given mixtures of secondary compounds. ACN and allyl isothiocyanate (AITC) were chosen as representatives of the two most prominent groups of glucosinolate breakdown products. DMDS was included as the other main brassica secondary compound. This simple threecomponent system was investigated to determine the potential physiological effects resulting from brassica consumption by ruminants.

\section{MATERIALS AND METHODS}

Animals and feed

Forty-four Scottish Blackface castrated male lambs (15 months old; mean live weight $37 \cdot 2$ (range 32-44) $\mathrm{kg}$ ) were housed at the beginning of June 1989. Animals were immediately introduced to a diet of dried grass pellets containing ( $\mathrm{g} / \mathrm{kg}$ dry matter (DM)) (N 23.9, neutral-detergent fibre (NDF) 549, acid-detergent fibre (ADF) 310, ash 81).

\section{Treatments and experimental design}

Animals were offered dried grass pellets $a d$ lib. and daily dry matter intakes were recorded for a $21 \mathrm{~d}$ pretreatment period. At the end of this period animals were ranked on the basis of VFI and the first forty in the rank order selected for the experiment. The rank order was divided into five blocks of eight animals each and treatments were randomly allocated to animals within blocks.

The experiment had a $2 \times 2 \times 2$ factorial design with levels consisting of presence or absence of each of three brassica secondary compound metabolites. Treatment factors 
Table 1. Stability of a mixture of dimethyl disulphide (DMDS), allyl cyanide $(A C N)$ and allyl isothiocyanate $(A I T C)$ with time

(Values are expressed as peak area as a proportion of internal standard peak area)

\begin{tabular}{cccc}
\hline Time $(\mathrm{h})$ & DMDS & ACN & AITC \\
\hline 0 & 0.871 & 0.800 & 0.890 \\
4 & 0.858 & 0.807 & 0.906 \\
69 & 0.806 & 0.791 & 0.901 \\
123 & 0.827 & 0.765 & 0.883 \\
144 & 0.881 & 0.792 & 0.894 \\
\hline
\end{tabular}

consisted of daily oral administration to each sheep for $35 \mathrm{~d}$ of $\mathrm{ACN}(0$ or $10 \mathrm{mmol} / \mathrm{d})$, AITC $(0$ or $10 \mathrm{mmol} / \mathrm{d})$ and DMDS $(0$ or $25 \mathrm{mmol} / \mathrm{d})$. All combinations of factors and levels yielded eight possible treatments.

A treatment period of 5 weeks was chosen to be of sufficient duration to allow development of anaemia as a result of DMDS administration (Smith, 1974), the rationale being that physiological interactions between glucosinolate and SMCO-derived compounds might not occur until anaemic symptoms had developed.

The higher level of each compound given was chosen on the basis of typical concentrations of brassica secondary compounds in cabbage and probable intakes of cabbage. For example, sinigrin concentrations in cabbage are in the region of $10 \mathrm{mmol} / \mathrm{kg}$ DM (Bradshaw et al. 1984) and wethers with a live weight of $40 \mathrm{~kg}$ might be expected to consume cabbage at the rate of $1 \mathrm{~kg} \mathrm{DM} / \mathrm{d}$. A dose rate of $10 \mathrm{mmol} \mathrm{ACN} / \mathrm{d}$ is, thus, equivalent to the potential in vivo release assuming complete hydrolysis of sinigrin to $\mathrm{ACN}$. Similar reasoning was applied in selecting dose rates of AITC and DMDS.

\section{Procedures}

Animals were given discrete doses of mixtures of the compounds under investigation twice daily. Animals were dosed with gelatin capsules containing the brassica secondary compounds at 08.00 and 16.00 hours throughout the treatment period.

Capsules were prepared in batches every $2-3 \mathrm{~d}$ by pipetting ACN (Aldrich, Gillingham, Dorset), AITC (Aldrich) and dimethyl disulphide (Sigma, Poole, Dorset) into gelatin capsules ( $3 \mathrm{ml}$; Davcaps, Monmouth, Gwent). Capsules were then capped and sealed with a strip of cellulose tape $(30 \times 10 \mathrm{~mm})$ ready for dosing.

The stability of mixtures of ACN, AITC and DMDS was verified by GLC before the experiment. An equimolar mixture of the three compounds was prepared from which portions were removed at intervals over $144 \mathrm{~h}$ and analysed on a Philips PU4400 gas-liquid chromatograph; at each sampling $15 \mathrm{mg}$ of the mixture was added to $5 \mathrm{ml}$ dichloromethane containing butanol as an internal standard $(1 \mathrm{mg} / \mathrm{ml})$ and $0.5 \mu 1$ of this solution was injected onto the GLC column. The column was a BP20 fused silica capillary column (SGE (UK) Ltd, Milton Keynes, Bucks; length $25 \mathrm{~m}$, i.d. $0.33 \mathrm{~mm}$, film thickness $1.0 \mu \mathrm{m}$ ); injection was in 'split mode' with an initial column temperature of $50^{\circ}$ which was maintained for $5 \mathrm{~min}$ before being increased at $5^{\circ} / \mathrm{min}$ to a final temperature of $120^{\circ}$. Peak areas were measured on a Philips PU4810 computing integrator and expressed relative to the internal standard. There was no indication of any reaction between compounds or degradation of compounds over $6 \mathrm{~d}$ (Table 1 ).

Daily VFI was measured as the difference between dry matter offered and refused with refusals being collected before each daily feed. Blood samples were collected from the 
jugular vein using evacuated heparinized tubes (Vacutainer, Becton Dickinson, Oxford, Oxon.). Concentrations of plasma triiodiothyronine (T3), plasma thyroxine (T4), blood haemoglobin, blood reduced glutathione, plasma albumin, plasma creatinine, plasma urea, activities of plasma $\gamma$-glutamyl transpeptidase ( $E C 2.3 .2 .1$; GGTP) and plasma alkaline phosphatase $(E C$ 3.1.3.1) together with Heinz body counts and blood packed cell volume (PCV) were measured weekly. Samples were collected on days 8 and 15 of the pretreatment period in order to establish baseline levels, and on days 2, 9, 16, 23 and 30 of the treatment period.

Urinary thiocyanate ion concentration and urinary $N$-acetyl-s-( $N$-allyl thiocarbamoyl)-Lcysteine (AATCC) concentration was measured in samples collected between 13.00 and 15.00 hours on day 17 of the treatment period. Urine samples were collected by placing a large clean polythene sheet under each pen for $2 \mathrm{~h}$ and collecting the run-off. Polyethylene bags were stapled into funnels at the lower end to allow urine collection and the resulting samples, which were relatively free of faecal contamination, were stored at $-20^{\circ}$ pending analysis.

Muscle cytochrome oxidase activity was measured in samples taken on day 25 of the treatment period. Muscle biopsies $(0.5 \mathrm{~g})$ were taken from the left hindlimb of each animal under general anaesthetic (Thiopentone injection followed by halothane $/ \mathrm{O}_{2}$ inhalation) between 08.00 and 12.00 hours (the 08.00 hours oral administration of compounds was withheld on the day of muscle sampling). The procedure was conducted under aseptic conditions and the animals were administered with a long-acting antibiotic after the procedure. Following excision, samples were immersed in ice-cold phosphate buffer $(0.05 \mathrm{M}, \mathrm{pH} 7.2)$ pending analysis for cytochrome oxidase activity.

\section{Measurements and analyses}

Plasma thyroid hormone analysis. Total plasma T3 and T4 concentrations were measured using Amerlex radioimmunoassay (RIA) kits (Amersham International, Bucks.). Free thyroxine index (FTI) was estimated from ' $T 3$ uptake' values which were determined using a commercial kit (T3 uptake (MAA) kit; Amersham International). The sensitivity of the T3 assay, calculated as the concentration which is two standard deviations above the zero standard, was $0.15 \mathrm{nmol} / 1$, while the within-assay coefficient of variation was $4 \%(n 50)$. The sensitivity of the T4 assay was $4.0 \mathrm{nmol} / 1$ and the within-assay coefficient of variation was $7 \%(n 56)$. The within-assay coefficient of variation for the T3 uptake test was $6 \%$ (n 19).

Liver and kidney function tests. Plasma albumin concentration was measured by the method of Spencer \& Price (1977), plasma creatinine concentration was analysed using the Jaffe reaction (Butler, 1975), and plasma urea concentration was determined using the Berthelot reaction (Wilcox et al. 1966). Plasma alkaline phosphatase activity was measured using a commercial diagnostic kit (Sigma procedure no. DG 1245) and plasma GGTP activity was measured by the method of Fuke et al. (1976).

Haematological measurements. Blood PCV was measured using the haematocrit method and blood haemoglobin was measured using a commercial kit (Sigma procedure no. 525). Blood reduced glutathione was measured by the method of Beutler et al. (1963). The percentage of erythrocytes containing Heinz bodies was determined on stained erythrocytes. For this purpose four drops of whole blood and four drops of Brilliant Cresyl Blue $(10 \mathrm{ml} / 1$ physiological saline $(9 \mathrm{~g} \mathrm{NaCl} / 1))$ were incubated in a test tube for $15 \mathrm{~min}$ at $37^{\circ}$. Blood smears were prepared using one drop of the previously described mixture and the resulting smears were examined using an oil-immersion microscope. Heinz bodies appeared as darkly staining granules at the periphery of the erythrocytes. The number of cells 
containing Heinz bodies was expressed as a percentage of the total number of cells counted $(>300)$.

Determination of cytochrome oxidase activity. Muscle cytochrome oxidase activity was measured within $6 \mathrm{~h}$ of tissue excision by the method of Cooperstein \& Lazarow (1951); activity was expressed relative to the protein content of the homogenates measured by the Lowry method (Sigma procedure no. P5656).

Analysis of ionic urinary thiocyanate. Urinary thiocyanate ion was measured by the method of Bowler (1944). The problem of non-specific absorbance due to urine pigments was avoided using the modification of Johnston \& Jones (1966). In brief, the absorbance of a coloured complex formed by the reaction of thiocyanate ion with $\mathrm{Fe}\left(\mathrm{NO}_{3}\right)_{3}$ was measured at $450 \mathrm{~nm}$. Duplicate tubes were prepared to which $10 \mu \mathrm{IgCl}_{2}(50 \mathrm{~g} / \mathrm{l}$; Sigma) was added to destroy the coloured complex. The difference in absorbance between the two tubes was taken as absorbance due to thiocyanate ion. Concentrations were calculated following construction of a calibration curve using KCNS standards.

Analysis of the urinary metabolite of AITC. Urine samples were analysed for the urinary metabolite of AITC, $N$-acetyl-S-( $N$-allylthiocarbamoyl)-L-cysteine (AATCC), by an HPLC method based on that of Mennicke et al. (1987). AATCC was synthesized for use as a quality control standard according to Mennicke et al. (1983) (melting point $141-145^{\circ}$; quoted range: $143-145^{\circ}$ ). Benzyl butyl thiourea (BBTU) was synthesized as described by Mennicke et al. (1987) for use as an internal standard.

Sample preparation was as follows. Urine $(1 \mathrm{ml})$ was added to a thick-walled, screw-top test tube $(150 \times 20 \mathrm{~mm}$ O.D., Philip Harris Scientific, London) along with $50 \mu \mathrm{l} n$ butylamine (Sigma) and $100 \mu \mathrm{l}$ BBTU internal standard in ethanol $(40 \mu \mathrm{g} \mathrm{BBTU} / \mathrm{ml}$ ethanol). Tubes were tightly capped with PTFE-lined screw tops and incubated in a waterbath at $60^{\circ}$ for $30 \mathrm{~min}$. When cool, $300 \mu \mathrm{H} \mathrm{H}_{2} \mathrm{SO}_{4}(250 \mathrm{ml} / \mathrm{l})$ was added to remove the excess amine. Following brief agitation the sample was extracted with $5 \mathrm{ml}$ diethyl ether (Aldrich). The diethyl ether extract was then washed sequentially with $1 \mathrm{ml} 1 \mathrm{M}-\mathrm{NaOH}$ and two washes with $1 \mathrm{ml}$ distilled water. A vacuum was then applied directly to the tube at room temperature using a water aspirator to evaporate the diethyl ether rapidly. The residue was dissolved in $1 \mathrm{ml}$ of the mobile phase, transferred to a glass HPLC vial and capped with a PTFE-lined crimp top.

HPLC separation was carried out on a Gilson modular HPLC system run in isocratic mode with a $250 \mathrm{~mm}$ Spherisorb S5ODS2 reverse-phase $C_{18}$ column (Phase Separations, Ltd, Clwyd). The mobile phase was water-acetonitrile (Aldrich) $(1: 1 ; \mathrm{v} / \mathrm{v})$ delivered at a flow-rate of $1 \mathrm{ml} / \mathrm{min}$. Injection volume was $20 \mu \mathrm{l}$ and detection was by u.v. absorbance at $240 \mathrm{~nm}$. Data were collected and peaks were integrated using standard 'Gilson 714' software on an IBM PC-AT personal computer. $N$-acetyl-S-( $N$-allylthiocarbamoyl)-Lcysteine concentration was calculated by comparing its peak area with that of the internal standard (BBTU) following calibration with synthetic AATCC standards. Quality control standards were analysed at ten-sample intervals throughout the analysis. These were prepared by dissolving $10 \mathrm{mg}$ AATCC in $10 \mathrm{ml}$ ethanol; $20 \mu \mathrm{l}$ of this solution was then added to $1 \mathrm{ml}$ distilled water and the resulting standards were treated identically to test urine samples in the analysis.

Feed analyses. Samples of feed offered and feed refused were taken daily, pooled by week and analysed for ash, total N, ADF and NDF. Ash content was determined by weighing residues after ashing in a furnace for $16 \mathrm{~h}$ at $450^{\circ}$. NDF was measured using the method of Van Soest \& Wine (1967) and ADF was determined by the method of Van Soest (1963). Total $\mathrm{N}$ was estimated by a semi-micro Kjeldahl block digestion method followed by a continuous flow method based on the Berthelot reaction (Alpkem Corp. RFA, method no. A303-S072; Wilsonville, Oregon, USA). 


\section{Statistical analysis}

Data were analysed by analysis of variance with error terms for blocks of animals, animals within blocks and weeks within animals. This assessed the main effects of DMDS, AITC, $\mathrm{ACN}$ and interactions between them using between-animal information. This also allowed a preliminary analysis of the effects of time and interactions with treatments using withinanimal variation. A more rigorous assessment of the effects of time was conducted by fitting linear regressions to data from individual animals over selected time-periods and conducting analysis of variance on the slopes using the between-animal terms of the model described previously (Rowell \& Walters, 1976). Analysis of variance was also conducted on data collected the week before the start of the treatments in order to verify the absence of effects before treatment application; of the thirty-nine main effects tested (13 variates, three treatments) only four showed significant effects at the $5 \%$ probability level and this was judged to be consistent with random variation existing before treatment application.

When analysing data relating to urinary metabolites of ACN (thiocyanate ion outputs) and AITC (AATCC outputs), only those animals receiving the precursor produced significant concentrations of the urinary metabolites. In each case, because half the animals produced essentially zero concentrations of the urinary metabolites, only animals receiving the administered precursor were included in statistical analyses of the urinary metabolite data.

All calculations used GENSTAT 5 (Lawes Agricultural Trust; Release 1.3, 1988).

\section{RESULTS}

Main effects

The main effects of dosing with DMDS, ACN and AITC on VFI, blood glutathione concentrations, anaemic status, thyroid hormone concentrations and a range of clinical diagnostic variables are shown in Table 2. These results refer to mean values over the whole treatment period, and the manner in which the variables changed with time is considered later.

$V F I$. VFI was significantly depressed by the presence of $\mathrm{ACN}(P<0.01)$ and AITC $(P<0.001)$ but not by the presence of DMDS.

Blood glutathione concentrations. The higher level of all three factors had highly significant effects on blood glutathione concentrations. The presence of $\mathrm{ACN}$ had the greatest effect in reducing mean blood-reduced glutathione concentrations which were 0.635 of those in the absence of $\operatorname{ACN}(P<0.001)$. A similar but less pronounced depression was caused by the presence of AITC with animals in this group having glutathione concentrations which were 0.825 of those in the absence of the compound $(P<0.001)$. Conversely, the presence of DMDS produced a rise in glutathione concentrations $(1.220$ of control values, $P<0.001$ ) when concentrations were averaged for the whole experiment.

Anaemic response. The extent of the anaemic response to treatments was relatively slight and neither haemoglobin concentrations nor PCV values were significantly altered by any of the treatments. Heinz body counts were elevated in the presence of DMDS $(P<0 \cdot 001)$.

Thyroid status. The presence of each factor tended to increase plasma concentrations of T3 and T4, although the effects of the presence of AITC and DMDS were not significant. The increase in T4 concentrations attained significance in the presence of $\mathrm{ACN}(P<0 \cdot 01)$; the presence of ACN also caused a significant increase in free thyroxine index (FTI) values $(P<0.05)$.

Liver and kidney function. Clinical indicators of liver and kidney function showed a number of significant treatment effects. Plasma albumin concentrations were slightly depressed in the presence of AITC $(P<0.05)$. Similarly, plasma urea concentrations were 
BRASSICA SECONDARY METABOLITES IN SHEEP

$\frac{2}{5}$

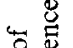

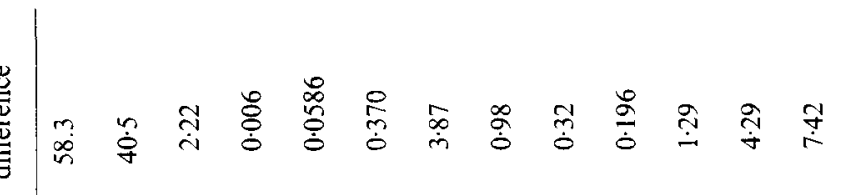
$\approx 0$ $\approx$ : 要过

害芯

$\vec{z} \approx$

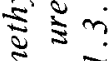

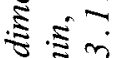

인

0 \%

공

$4 \frac{5}{2}$

ปั่

ฐ 5

$\stackrel{2}{\pi}$

20

. 3

言 $\hat{\vec{\alpha}} \frac{\bar{s}}{0}$

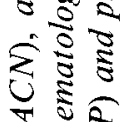

¿

넌

ป

o 5

$3 \pm$

จ

ธ.

胥

氖象

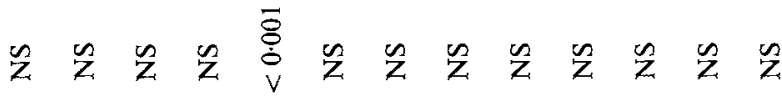

Q

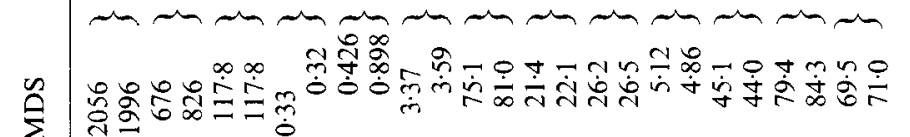

$\sum_{0}$

$1+1+1+1+1+1+1+1+1+1+1+1+$

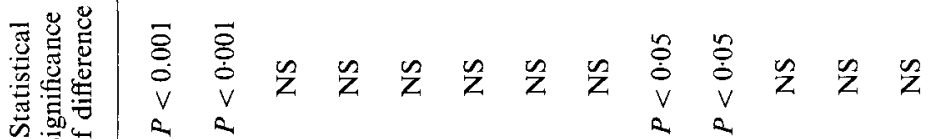

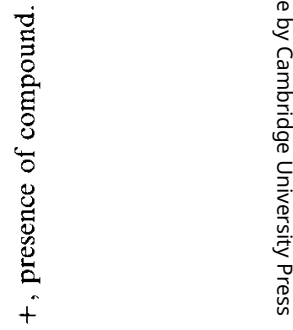

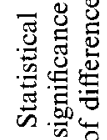

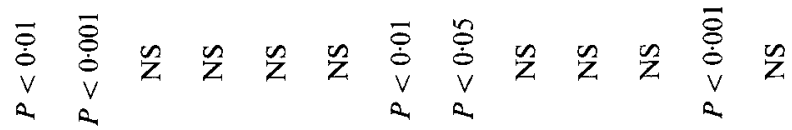

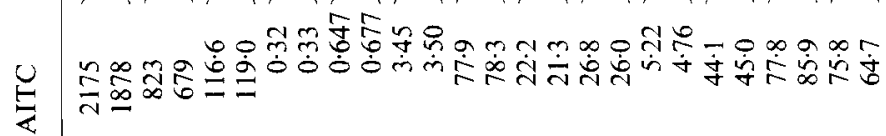

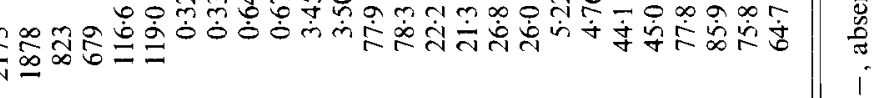

$1+1+1+1+1+1+1+1+1+1+1+1+1+$

节

$2 \quad 2$

ถิ

$\tilde{z} \underset{\mathrm{c}}{\mathrm{N}}$

苍完

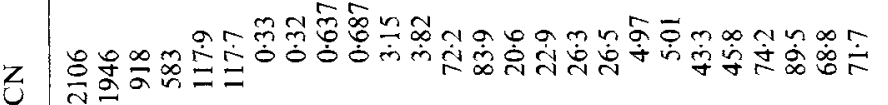

要

४

$1+1+1+1+1+1+1+1+1+1+1+1+1+$

通常

$\stackrel{3}{3}$

$\stackrel{5}{5}$

-

ป⿱艹

$\approx 5$

ธิ

0 紊

$5: \frac{5}{\infty}$

¿ँ

넌

त है है

$\simeq \approx$

है

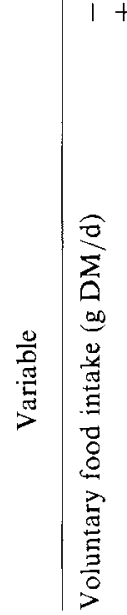

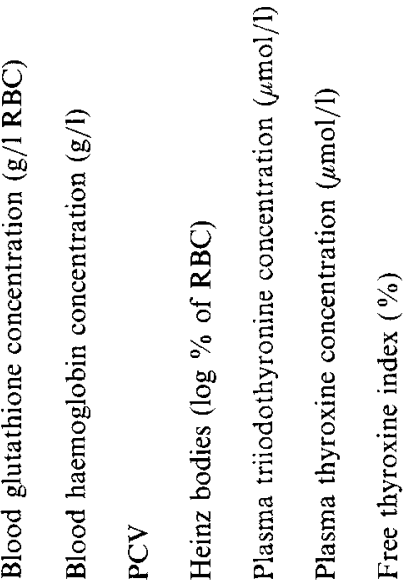
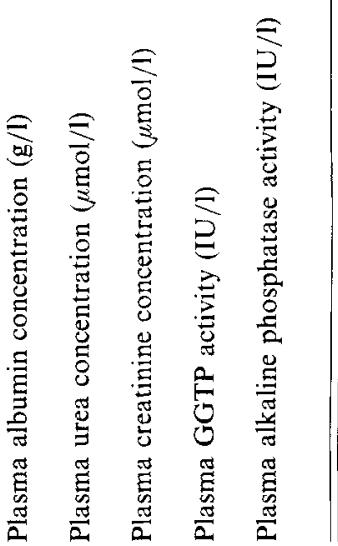

记

is

롤

उप्ष

这 
Table 3. Two-way interactions between allyl cyanide $(A C N)$, allyl isothiocyanate (AITC) and dimethyl disulphide (DMDS); effects on voluntary food intake and blood packed cell volume of sheep*

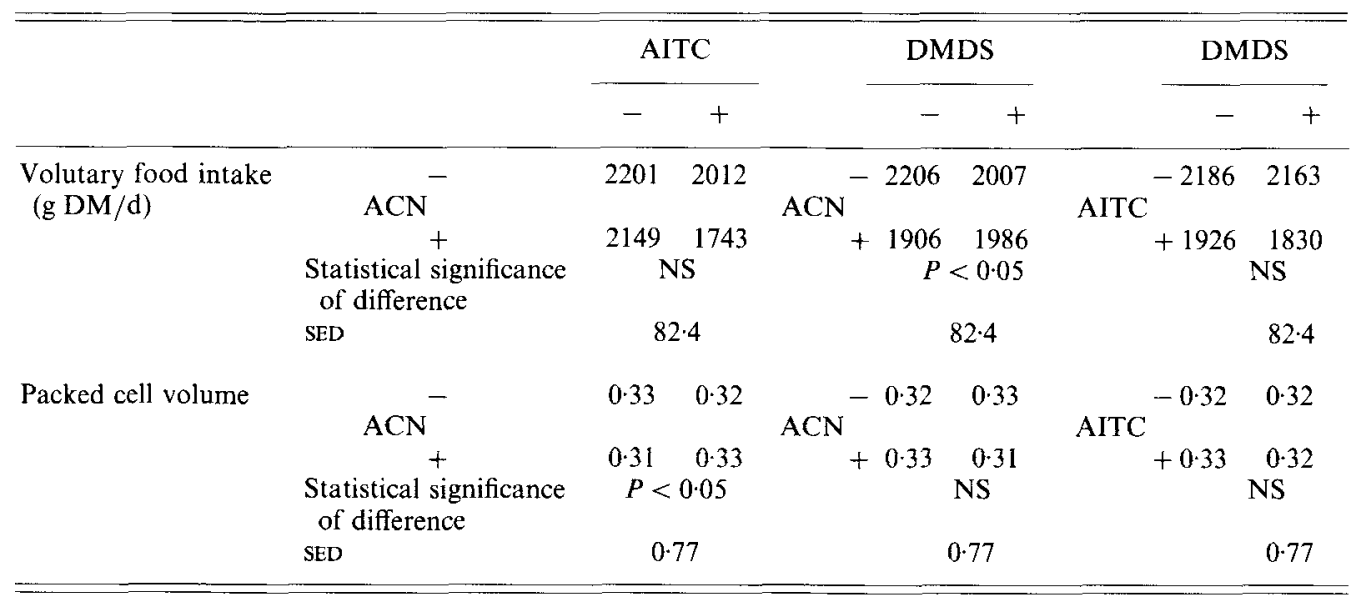

DM, dry matter; SED, SE of difference; NS, not significant; - , absence of compound; + , presence of compound.

* For details of procedures, see pp. 632-636.

depressed when AITC was present $(P<0.05)$. Plasma creatinine concentrations and plasma alkaline phosphatase activity were unaffected by treatment. Plasma GGTP activity was significantly elevated in the presence of ACN $(P<0.001)$; a similar, but less pronounced, effect which approached significance $(P=0.067)$ was observed when AITC was present.

Cytochrome oxidase activity. Muscle cytochrome oxidase activity measured on day 25 of the treatment period was not significantly altered by the presence or absence of any of the three compounds administered.

Urinary metabolite concentrations. Both AITC and ACN had large effects on the concentrations of their respective metabolites in the urine. Thus, the presence of AITC resulted in mean urinary concentrations of AATCC of 29.5 (SE 4.94$) \mu \mathrm{g} / \mathrm{ml}$, while in its absence the mean concentration of AATCC was 0.9 (SE 0.30) $\mu \mathrm{g} / \mathrm{ml}$. Similarly, the mean urinary concentration of thiocyanate ions was $8.73(\mathrm{SE} 1.250) \mu \mathrm{g} / \mathrm{ml}$ in the presence of $\mathrm{ACN}$ but only $0.32(\mathrm{SE} 0.225) \mu \mathrm{g} / \mathrm{ml}$ in its absence.

\section{Interaction effects}

There was a significant interaction between the effects of ACN and DMDS on VFI $(P<0.05)$, whereby the presence of each compound singly caused a reduction in VFI but the extent of the reduction caused by the presence of the two compounds in combination was less than the sum of their individual effects (Table 3).

There was a significant interaction between the effects of ACN and AITC on PCV values (Table 3). ACN marginally depressed PCV values in the absence of AITC, while in the presence of AITC the values were slightly elevated (PCV, $P<0.05$ ). The only significant three-way interaction was with respect to albumin concentrations; in the absence of $\mathrm{ACN}$ albumin concentrations were elevated by DMDS and depressed by AITC, while in the presence of $\mathrm{ACN}$ the converse applied $(P<0.05)$.

Catabolism of $\mathrm{ACN}$ to urinary thiocyanate ions was affected by the additional presence 

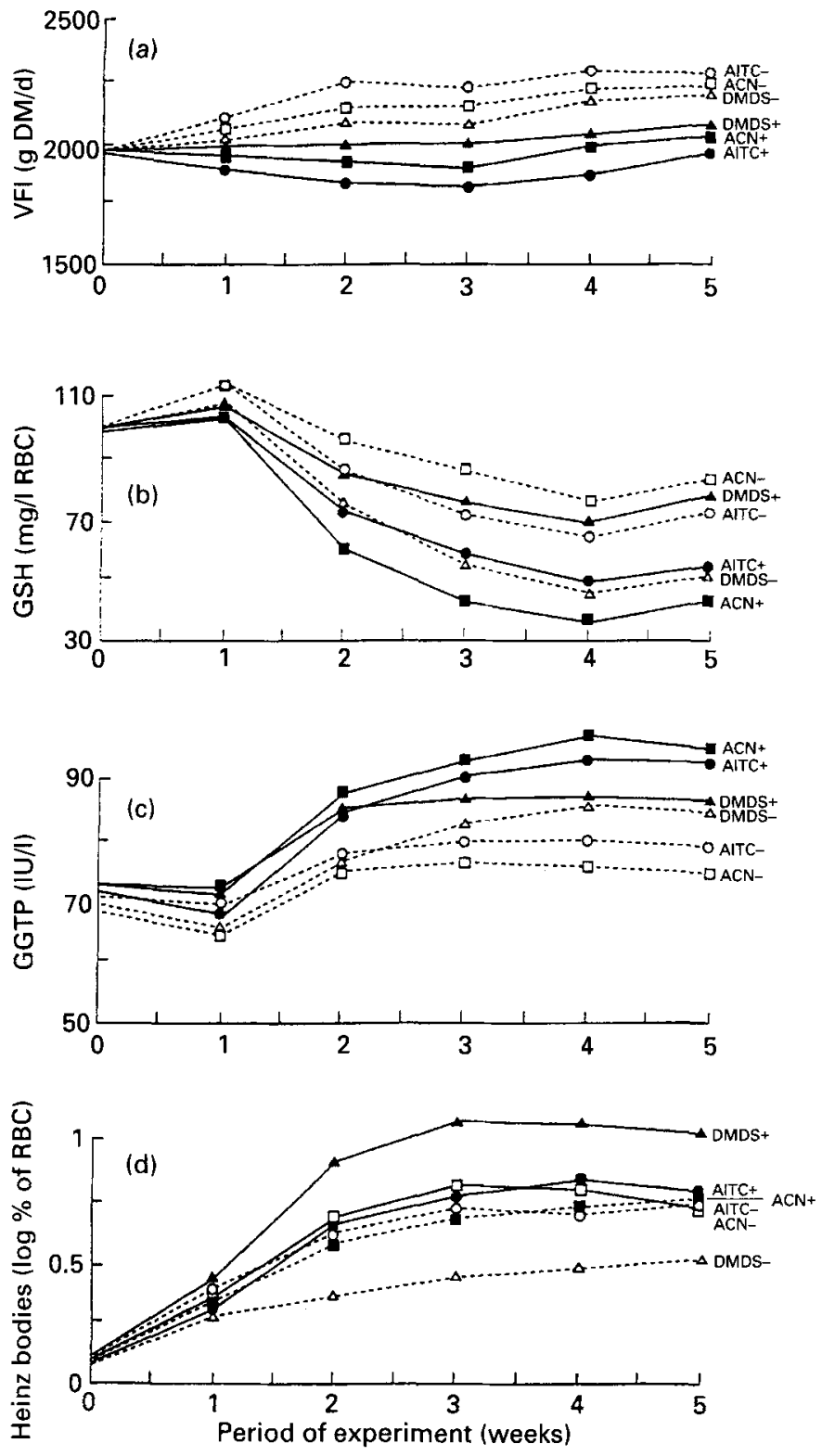

Fig. 1. Changes with time of the main effects of daily administration to sheep of allyl cyanide ( $10 \mathrm{mmol} / \mathrm{d} ; \mathrm{ACN}$; $\square, \square)$, allyl isothiocyanate ( $10 \mathrm{mmol} /$ day; AITC; $\bigcirc$, ), and dimethyl disulphide $(25 \mathrm{mmol} / \mathrm{d} ; \mathrm{DMDS} ; \triangle, \mathbf{\Delta})$ on (a) voluntary food intake (g DM/d; VFI), (b) blood-reduced glutathione (GSH) concentration (mg/1 erythrocytes (RBC)), (c) plasma $\gamma$-glutamyl transpeptidase (EC 2.3.2.1; GGTP) activity (IU/1) and (d) Heinz body count ( $\log (\%$ RBC containing Heinz bodies)). $\square, O, \triangle$, In the absence of each compound; $\boldsymbol{\square}, \boldsymbol{O}, \boldsymbol{\Delta}$, in the presence of each compound. Because of the factorial nature of the experimental design, each line represents the mean of half the experimental units; i.e. $n 20$ for each line). For deatils of procedures, see pp. 632-635.

of AITC or DMDS. The presence of AITC significantly increased the urinary concentration of thiocyanate ion when compared with concentrations in the absence of AITC (AITC present, $11.43 \mu \mathrm{g} / \mathrm{ml}$; AITC absent, $5.55 \mu \mathrm{g} / \mathrm{ml}$; SE of difference (SED) $1.433 ; P<0.005$ ). Conversely, the urinary thiocyanate ion concentration in the presence of DMDS was lower 
than in its absence (DMDS present, $6.59 \mu \mathrm{g} / \mathrm{ml}$; DMDS absent, 10.39 $\mu \mathrm{g} / \mathrm{ml}$; SED 1.433; $P<0.05$ ). Catabolism of AITC to urinary AATCC on the other hand was not significantly affected by the additional presence of $\mathrm{ACN}$ or DMDS.

\section{Effects of time}

Weekly measurements made over the 5 -week treatment period enabled investigation of the extent to which responses to the treatments developed over time. Changes in VFI, blood reduced glutathione concentrations, plasma GGTP activities and Heinz body counts with time are presented in Fig. 1.

VFI declined during the first 2 weeks in response to the presence of AITC (ANOVA of slopes, $P<0.001$ for change from week 0 to week 2) and ACN (ANOVA of slopes, $P<0.01$ for change from week 0 to week 2). VFI did not change significantly after week 2 (ANOVA of slopes; AITC, $P<0.05$ for difference in slopes from week 2 to week 5 ; ACN, $P>0.05$ for difference in slopes from week 2 to week 5 ).

In the presence of DMDS, blood-reduced glutathione concentrations were higher than those in the absence of DMDS, with a steady increase occurring over the 5-week period (ANOVA of slopes, $P<0.001$ ). The presence of ACN and AITC, however, caused a reduction in blood-reduced glutathione concentrations with most of the change occurring during weeks 1-3 (ANOVA of slopes; ACN, $P<0.001$; AITC, $P<0.001$ ).

Anaemic variables showed some evidence of a change with time. The increase in Heinz body count in the presence of DMDS occurred chiefly during the first 2 weeks of the period (ANOVA of slopes, $P<0.001$ ) and differences in Heinz body numbers due to the presence of the compound remained fairly constant thereafter. $\mathrm{PCV}$ values of $\mathrm{ACN}$-treated animals decreased relative to controls over the first 4 weeks of the treatment period (changing from 33.70 to $32.08 \%$ between weeks 1 and 4 in the absence of ACN compared with a change from 34.66 to $30 \cdot 17 \%$ over the same period in the presence of ACN; ANOVA of slopes, $P<0.001)$.

Of the three variables associated with thyroid status (plasma T3 concentration, plasma T4 concentration and free T4 index) the only significant effect of time was with plasma T3 concentrations in ACN-treated animals. Most of the effect of ACN on plasma T3 concentrations occurred between weeks 1 and 2 of the treatment period (changing from 4.206 to $3.105 \mu \mathrm{mol} / 1$ between weeks 1 and 2 in the absence of ACN compared with a change from 3.730 to $4.019 \mu \mathrm{mol} / 1$ over the same period in the presence of ACN; ANOVA of slopes, $P<0.05$ ).

Among clinical indicators of liver and kidney function, plasma GGTP activity showed significant changes with time; the largest increase in plasma GGTP activity occurred between weeks 1 and 2 in the presence of ACN (ANOVA of slopes, $P<0.05$ ) and also in the presence of AITC (ANOVA of slopes, $P<0 \cdot 01$ ) (Fig. 1).

\section{DISCUSSION \\ Effects of $A C N$}

In agreement with another experiment on the effects of long-term infusion of $A C N$ (Duncan \& Milne, 1992), ACN caused a significant depression of VFI in the present experiment. The possibility that ACN was acting directly on micro-organisms in the rumen by reducing their activity and so reducing VFI via reduced digestibility and rate of passage cannot be excluded. Such effects of plant secondary compounds on rumen microflora have been demonstrated previously, for example, with phenolic acids (Martin, 1988; Hartley \& Akin, 1989) and terpenoid compounds (Oh et al. 1967). However, the most probable mechanism for toxicity of nitriles involves inhibition of aerobic respiration following 
release of free cyanide (Willhite \& Smith, 1981; Tanii \& Hashimoto, 1984) which suggests that the toxicity of ACN is more likely to be manifested systemically than in the anaerobic environment of the rumen.

Systemic effects were apparent; among clinical indicators of liver and kidney function, plasma GGTP activity was elevated by ACN, indicating liver damage to have been caused by the compound, although plasma albumin concentrations and plasma alkaline phosphatase activity were unaffected by treatment. $\gamma$-Glutamyl transpeptidase is a more sensitive indicator of liver damage than alkaline phosphatase and is also specific to liver tissues. Plasma albumin concentrations are only depressed in cases of advanced liver necrosis. This evidence of liver damage caused by a glucosinolate-derived nitrile is in agreement with studies with single-stomached animals (Pearson et al. 1979; Campbell, 1987).

ACN administration caused a dramatic reduction in blood-reduced glutathione concentrations. The effects of nitriles and other electrophilic compounds in reducing glutathione concentrations of other tissues, and in particular liver tissues, are well known (Orrenius \& Moldeus, 1984) but the parallel depletion in erythrocytes has not been demonstrated previously.

Release of free cyanide in the liver from nitriles and subsequent metabolism of cyanide to thiocyanate ions in the liver by thiosulphate sulphurtransferase (EC 2.8.1.1) would be expected to increase concentrations of circulating thiocyanate ions whose goitrogenic properties have previously been demonstrated (Paxman \& Hill, 1974). Thyroid function, as measured by plasma concentrations of thyroid hormones, did not, however, appear to be depressed by ACN. Indeed, peripheral T4 levels were elevated in the presence of ACN. Recent work has demonstrated the role of reduced glutathione in the de-iodination of T4 to $\mathrm{T} 3$ in rats with glutathione depletion leading to a build-up of circulating T4 (Suberville et al. 1988). Thus, ACN may have had an indirect effect on T4 concentrations through its action as a glutathione depletor.

\section{Effects of AITC}

The intake depression caused by oral administration of AITC suggests a possible role for AITC as a factor contributing to the lower than predicted performance of sheep offered forage brassicas (see Milne, 1990). The mechanism of VFI depression did not appear to be associated with the previously described goitrogenicity of the isothiocyanates, since peripheral thyroid hormone concentrations were unaffected by AITC administration.

In common with ACN, AITC caused severe depletion of blood-reduced glutathione concentrations, although the significance of this effect in relation to reduced VFI is unclear. Blood-reduced glutathione concentrations appeared to be sufficient to maintain protection in the erythrocytes against peroxide and singlet oxygen toxicity since there was no evidence of haemolysis and anaemia in the presence of AITC. Kidney function did not appear to be affected by AITC since neither plasma creatinine nor urea concentrations were elevated in the presence of the compound. Reduced plasma concentrations of albumin and increased plasma GGTP activity provided some indication of effects on liver metabolism, although the effects were less marked than with ACN.

The possibility that AITC exerted its effects in the digestive tract itself was not investigated but cannot be excluded. A number of potential modes of action can be conceived including effects on rumen motility, inhibition of microbial activity, or direct inhibition of digestive enzymes. In vitro studies have demonstrated the anti-bacterial action of isothiocyanates (Drobnica, 1967), and their capacity to form conjugates with proteins including digestive enzymes (Tang, 1974), and the importance of these processes in vivo requires clarification. 


\section{Effects of DMDS}

DMDS did not affect VFI at the level tested in this experiment. DMDS has been shown to cause haemolytic anaemia in previous studies (Smith, 1974). The only measurement associated with haemolytic anaemia to be affected by the treatment was the percentage of erythrocytes containing Heinz bodies. Heinz body numbers in the presence of DMDS (10-15\% of erythrocytes) indicated a mild anaemic effect; by comparison, typical levels produced as a result of the haemolytic anaemia caused by brassica ingestion would be 30-50\% (Greenhalgh et al. 1970). Since PCV levels and blood haemoglobin concentrations were unaffected by DMDS, the production of Heinz bodies in the erythrocytes was apparently not sufficient to bring about haemolysis and anaemia.

The elevation in blood-reduced glutathione concentrations as a result of DMDS administration conflicts with previous studies in which feeding of whole kale to ruminants has resulted in a decline in blood-reduced glutathione concentrations (Barry et al. 1982). By separating the effects of different brassica secondary compounds the present experiment indicates the decline in blood-reduced glutathione concentration during brassica feeding to be due to the glucosinolate component of brassicas. Glutathione concentrations decrease with cell age (Young \& Tucker, 1983) and replacement of older susceptible cells with younger, glutathione-rich cells in response to anaemia would have the effect of elevating mean blood-reduced glutathione concentrations. Further studies could quantify the rate of erythrocyte recruitment during brassica-induced anaemia to determine whether DMDS increases reduced glutathione concentrations in existing cells or whether the increased mean blood-reduced glutathione concentration observed is a result of increased erythrocyte turnover.

\section{Effects of time}

Consideration of the time-course of development of physiological effects is potentially useful in correlating VFI depression with underlying biochemical events. The effects of ACN in reducing VFI were most pronounced in weeks 1 and 2 of the treatment period. Similarly, changes in other biochemical variables were most marked during this period and, thus, it is difficult to correlate effects on VFI with any single variable. Changes in VFI due to the AITC treatment were also greatest in the first 2 weeks of the treatment period. Conversely, effects on the haematological variables occurred later in the treatment period, casting doubt on their importance in relation to VFI depression.

\section{Interaction effects}

Previous infusion experiments in which glucosinolate breakdown products were infused into lambs fed on forage rape or dried grass pellets indicated that interactions between glucosinolates and other components of the brassica diet might be important in dictating their effects on the animal (Duncan, 1990). Thus, infusion of ACN $(2.4 \mathrm{mmol} / \mathrm{d}$ ) into lambs offered forage rape caused a reduction in VFI, while infusion at a higher rate $(4.8 \mathrm{mmol} / \mathrm{d})$ into grass-fed lambs had no effect. Furthermore, plasma T4 concentrations were significantly depressed by AITC infusion in rape-fed lambs but not in grass-fed lambs (Duncan, 1990). The other prominent secondary compound found in the vegetative material of brassica species is SMCO. The physiological effects of secondary plant compounds may be influenced by levels of macro-nutrients (Kappas et al. 1977) and the presence of other groups of secondary compounds (Scofield et al. 1990) in the diet. This potential for interactions between separate groups of secondary plant compounds is particularly pertinent in brassicas which contain two separate groups of secondary compounds. Interactions would be hypothesized to occur as a result of the anaemia caused by DMDS predisposing to more severe detrimental effects of glucosinolates. Since the 
extent of the anaemia resulting from DMDS administration was slight in comparison to that seen in animals grazing forage brassica crops, such an interaction was not tested under extreme conditions in the present experiment. However, other interactions between glucosinolate breakdown products and DMDS were found, particularly in relation to their metabolic fate. For example, thiocyanate ion concentrations in the urine of animals given ACN were decreased by the additional presence of DMDS, indicating cyanide release to be decreased by the presence of DMDS. Conversely, the presence of AITC increased urinary thiocyanate ion excretion by animals given $\mathrm{ACN}$, indicating increased cyanide production in the tissues. Interestingly, parallel interactions were apparent with respect to VFI. Thus, reduced free cyanide release in the presence of DMDS was accompanied by less marked effects of the presence of ACN on VFI when DMDS was also present. Examination of interactions among the other physiological variables measured provided little evidence for more pronounced effects as a result of the combined administration of compounds. Such effects might have been more apparent had the haemolytic anaemia caused by DMDS been more severe.

The present experiment has shown glucosinolate breakdown products to cause a depression in VFI of sheep. The marked blood-reduced glutathione depletion caused by both glucosinolate breakdown products did not exaberate the effects of DMDS in causing haemolytic anaemia. However, because the infusion levels of DMDS did not produce anaemia of the magnitude observed in animals consuming forage brassica herbage in the field, the potential importance of such interactions cannot be ruled out. Furthermore, changes in the magnitude of effects which resulted from the combined administration of brassica secondary compounds and which could be correlated with altered metabolic fate highlighted the importance of considering all components of a diet in combination before judging the harmful potential of an isolated constituent. As clinical diagnostic tests indicated that both ACN and AITC caused cellular damage to liver tissues, future work on the effects of glucosinolate should concentrate on liver function.

Thanks to Dr G. R. Iason for useful comments on the manuscript, to Dr P. Goddard (MRCVS) for conducting the muscle biopsies and to Mr D. A. Elston (Scottish Agricultural Statistics Service) for statistical advice. Thanks also to Messrs. J. Leary and G. Bryce for care and feeding of the animals and to Mr R. Fawcett for advice on animal health.

\section{REFERENCES}

Ahmed, P. \& Farooqui, M. Y. H. (1982). Comparative toxicities of aliphatic nitriles. Toxicology Letters 12 , $157-163$.

Barry, T. N., Manley, T. R. \& Millar, K. R. (1982). Nutritional evaluation of kale diets. 4. Responses to supplementation with synthetic S-methyl cysteine sulphoxide (SMCO). Journal of Agricultural Science, Cambridge 99, 1-12.

Beutler, E., Duran, O. \& Kelly, B. M. (1963). Improved method for the determination of blood glutathione. Journal of Laboratory and Clinical Medicine 61, 882-888.

Bjorkman, R. (1973). Interaction between proteins and glucosinolate isothiocyanate and oxazolidinethiones from B. napus seed. Phytochemistry 12, 1585-1590.

Bowler, R. G. (1944). The determination of thiocyanate in blood serum. Biochemical Journal 38, 385-388.

Bradshaw, J. E., Heaney, R. K., Macfarlane Smith, W. H., Gowers, S., Gemmel, D. J. \& Fenwick, G. R. (1984). The glucosinolate content of some fodder brassicas. Journal of the Science of Food and Agriculture 35, 977-981.

Butler, A. R. (1975). The Jaffe reaction. Identification of the coloured species. Clinica Chimica Acta 59, $227-232$.

Campbell, L. D. (1987). Intact glucosinolates and glucosinolate hydrolysis products as causative agents in liver hemorrhage in laying hens. Nutrition Reports International 36, 491-496.

Cooperstein, S. J. \& Lazarow, A. (1951). A microspectrophotometric method for the determination of cytochrome oxidase. Journal of Biological Chemistry 189, 665-670.

Drobnica, L. (1967). Wirkungsmechanismus einiger Isothiocyanate auf Hefen und Bakterien. [Mechanical effects 
of some isothiocyanates with yeasts and bacteria]. In Mechanisms of Action of Fungicides and Antibiotics, pp. 131-139 [M. Girbardt, editor]. Berlin: Akademie-Verlag.

Duncan, A. J. (1990). The physiological effects of glucosinolates and S-methyl cysteine sulphoxide on sheep consuming forage brassica crops. PhD Thesis, University of Edinburgh.

Duncan, A. J. \& Milne, J. A. (1992). Effect of long-term intra-ruminal infusion of the glucosinolate metabolite allyl cyanide on the voluntary food intake and metabolism of lambs. Journal of the Science of Food and Agriculture 58, 9-14.

Fenwick, G. R. \& Heaney, R. K. (1983). Glucosinolates and their breakdown products in cruciferous crops, foods and feeding stuffs. Food Chemistry 11, 249-271.

Fuke, H., Yagi, H., Takegoshi, C. \& Kondo, T. (1976). A sensitive automated colorimetric method for the determination of serum gamma-glutamyl transpeptidase. Clinica Chimica Acta 69, 43-51.

Gordon-Smith, E. C. \& White, J. M. (1974). Oxidative haemolysis and Heinz body anaemia. British Journal of Haematology 26, 513-517.

Gould, D. H., Fettman, M. J., Daxenbichler, M. E. \& Bartuska, B. M. (1985). Functional and structural alterations of the rat kidney induced by the naturally occurring organonitrile, 2S-1-cyano-2-hydroxy-3, 4epithiobutane. Toxicology and Applied Pharmacology 78, 190-201.

Greenhalgh, J. F. D., Sharman, G. A. M. \& Aitken, J. N. (1970). Kale anaemia. II. Further factors concerned in the occurrence of the disease under experimental conditions. Research in Veterinary Science 11, 232-238.

Hartley, R. D. \& Akin, D. E. (1989). Effect of forage cell wall phenolic acids and derivatives on rumen microflora. Journal of the Science of Food and Agriculture 49, 405-411.

Johnston, T. D. \& Jones, D. I. H. (1966), Variation in the thiocyanate content of kale varieties. Journal of the Science of Food and Agriculture 17, 70-71.

Kappas, A., Anderson, K. E., Conney, A. H. \& Alvares, A. P. (1977). Influence of dietary protein and carbohydrate on antipyrine and theophylline metabolism in man. Clinical Pharmacology and Therapeutic's 20, 643-653.

Kawakishi, S., Goto, T. \& Namiki, M. (1983). Oxidative scission of the disulphide bond of cystine and polypeptides by the action of allyl isothiocyanate. Agricultural and Biological Chemistry 47, 2071-2076.

Kawakishi, S. \& Namiki, M. (1982). Oxidative cleavage of the disulfide bond of cystine by allyl isothiocyanate. Journal of Agricultural and Food Chemistry 30, 618-620.

Langer, P. (1964). Study of the chemical representatives of the goitrogenic activity of raw cabbage. Physiologia Bohemoslovenica 3, 542-549.

Langer, P. \& Stolc, V. (1965). Goitrogenic activity of allylisothiocyanate - a widespread natural mustard oil. Endocrinology 76, 151-155.

Lawes Agricultural Trust (1988). Genstat 5, Release 1.3. Harpenden, Herts: Rothamsted Experimental Station.

Martin, S. A. (1988). Effects of $p$-coumaric acid and ferulic acid on methane production and fibre digestion by mixed rumen micro-organisms in vitro. Letters in Applied Microbiology 7, 113-114.

Mennicke, W. H., Gorler, K. \& Krumbiegel, G. (1983). Metabolism of some naturally occurring isothiocyanates in the rat. Xenobiotica 13, 203-207.

Mennicke, W. H., Kral, T., Krumbiegel, G. \& Rittman, N. (1987). Determination of $N$-acetyl-S- $(N-$ alkylthiocarbamoyl)-L-cysteine, a principal metabolite of alkyl isothiocyanates in rat urine. Journal of Chromatography 414, 19-24.

Milne, J. A. (1990). Brassica leaf and root crops - a review of research findings in relation to animal production. In Milk and Meat from Forage Crops. British Grassland Society Occasional Publication no. 24 [G. E. Pollot, editor]. Maidenhead, Berkshire: British Grassland Society.

Nishie, K. \& Daxenbichler, M. E. (1980). Toxicology of glucosinolates, related compounds (nitriles, R-goitrin, isothiocyanates) and vitamin U found in Cruciferae. Food and Cosmetic Toxicology 18, 159-172.

Oh, H. K., Sakai, T., Jones, M. B. \& Longhurst, W. M. (1967). Effect of various essential oils isolated from Douglas Fir needles upon sheep and deer rumen microbial activity. Appled Microbiology 15, 777-784.

Ohkawa, H., Ohkawa, R., Yamamoto, I. \& Casida, J. E. (1972). Enzymatic mechanisms and toxicological significance of hydrogen cyanide liberation from various organothiocyanates and organonitriles in mice and houseflies. Pesticide Biochemistry and Physiology 2, 95-112.

Orrenius, S. \& Moldeus, P. (1984). The multiple roles of glutathione in drug metabolism. Trends in Pharmacological Science 5, 432-435.

Paxman, P. J. \& Hill, R. (1974). The goitrogenicity of kale and its relation to thiocyanate content. Journal of the Science of Food and Agriculture 25, 329-337.

Pearson, A. W., Butler, E. J. \& Fenwick, G. R. (1979). Rapeseed meal and liver damage: effect on plasma enzyme activity in chicks. Veterinary Record 105, 200-201.

Rowell, J. G. \& Walters, D. E. (1976). Analysing data with repeated observations on each experimental unit. Journal of Agricultural Science, Cambridge 87, 423-432.

Scofield, A. M., Rossiter, J. T., Witham, P., Kite, G. C. \& Nash, R. J. (1990). Inhibition of thioglucosidecatalysed glucosinolate hydrolysis by castanospermine and related alkaloids. Phytochemistry 29, 107-109.

Silver, E. H., Kuttab, S. H. \& Hasan, T. (1982). Structural considerations in the metabolism of nitriles to cyanide in vivo. Drug Metabolism and Disposition 10, 495-498.

Smith, R. H. (1974). Kale poisoning. Report of the Rowett Research Institute 30, 112-131. 
Smith, R. H. (1980). Kale poisoning: the brassica anaemia factor. Veterinary Record 107, 12-15.

Spencer, K. \& Price, C. P. (1977). Influence of reagent quality and reaction conditions on determination of serum albumin by the bromo-cresol dye-binding method. Annals of Clinical Biochemstry 14, 105-115.

Srivastava, V. K., Philbrick, D. J. \& Hill, D. C. (1975). Responses of rats and chicks to rapeseed meal subjected to different enzymatic treatments. Canadian Journal of Animal Science 55, 331-335.

Steven, F. S., Griffin, M. M. \& Smith, R. H. (1981). Dimethyl exchange reactions in the control of enzymic activity. Evidence for the participation of dimethyl disulphide in exchanges. European Journal of Biochemistry 119, 75-78.

Suberville, C., Higueret, P., Taruaoura, D., Garcin, H. \& Higueret, D. (1988). Glutathione deficiency and peripheral metabolism of thyroid hormones during dietary cysteine deprivation in rats. British Journal of Nutrition 59, 451-456.

Tang, C. S. (1974). Benzyl isothiocyanate as a naturally occurring papain inhibitor. Journal of Food Science 39 , $94-96$.

Tanii, H. \& Hashimoto, K. (1984). Studies on the mechanism of acute toxicity of nitriles in mice. Archives of Toxicology 55, 47-54.

VanEtten, C. H., Gagne, W. E., Robbins, D. J., Booth, A. N. \& Daxenbichler, M. E. (1969). Biological evaluation of crambe seed meals and derived products by rat feeding. Cereal Chemistry 46, 145-155.

Van Soest, P. J. (1963). Use of detergents in the analysis of fibrous feeds. II. A rapid method for the determination of fiber and lignin. Journal of the Association of Official Analytical Chemists 46, 829-835.

Van Soest, P. J. \& Wine, R. H. (1967). Use of detergents in the analysis of fibrous feeds. IV. Determination of plant cell-wall constituents. Journal of the Association of Official Analytical Chemists 50, 50-55.

Wilcox, A. A., Coroll, W. E., Sterling, R. E., Davis, H. A. \& Ware, A. G. (1966). Use of the Berthelot reaction in the automated analysis of serum urea nitrogen. Clinical Chemistry 12, 151-157.

Willhite, C. C. \& Smith, R. P. (1981). The role of cyanide liberation in the acute toxicity of aliphatic nitriles. Toxicology and Applied Pharmacology 59, 589-602.

Young, J. D. \& Tucker, E. M. (1983). Erythrocyte glutathione deficiency in sheep. In Functions of Glutathione: Physiological, Toxicologic [A. Larsson, S. Orrenius, A. Holmgren and B. Mannervik, editors]. New York: Raven Press. 